\title{
REPRESENTATION OF $A$-CONVEX ALGEBRAS
}

\author{
ALLAN C. COCHRAN
}

\begin{abstract}
Algebraic properties of $A$-convex algebras are developed via a functor to locally $m$-convex algebras. The Gel'fandMazur theorem holds for $A$-convex algebras, and this fact allows a Gel'fand-type representation theorem for a subclass of uniformly $A$-convex algebras. Connections to existing functional representation theory are also obtained.
\end{abstract}

1. Introduction. The objects of this note are to give some algebraic properties of $A$-convex algebras, to note the fact that the Gel'fand-Mazur theorem holds for this class of algebras and to develop a Gel'fand-type theorem appropriate for certain $A$-convex algebras. The main technique for obtaining algebraic properties is to use a functor from the category of $A$-convex algebras to the category of locally $m$-convex algebras and then use the existing theory of $m$-convex algebras (see, for example, [5]). Various relations are developed along these lines.

The fact that the Gel'fand-Mazur theorem holds for $A$-convex algebras means that for commutative algebras there is a one-to-one correspondence between continuous nonzero multiplicative linear functionals and the closed regular maximal ideals. However, the generalizations of the Gel'fand representation theory on this carrier space (e.g. [6]) are all in terms of a locally $m$-convex algebra. We develop a representation theorem for a subclass of $A$-convex algebras (uniformly $A$-convex) which gives the $m$-convex results as corollaries. A type of strict topology is used on the space of continuous functions in which the algebra is embedded.

Basic results on $A$-convex algebras are found in [1], [2] and [3]. In order to make this note relatively self-contained we briefly repeat pertinent definitions. A locally convex algebra is an algebra over $\boldsymbol{R}$ or $\boldsymbol{C}$ with a locally convex topology for which multiplication is separately continuous. An $A$-convex seminorm on an algebra $E$ is a seminorm $p$ such that for $x \in E$ there are constants $M_{x}$ and $N_{x}$ such that $p(x y) \leqq M_{x} p(y)$ and $p(y x) \leqq$ $N_{x} p(y)$ for all $y \in E$. An A-convex algebra \{locally m-convex algebra\}

Received by the editors March 15, 1973.

AMS (MOS) subject classifications (1970). Primary 46H15; Secondary :6H05, $46 \mathrm{H} 20$.

Key words and phrases. A-convex algebra, locally $m$-convex algebra, Gel'fand-Mazur theorem, strict topology, compact-open topology, Gel'fand representation theorem.

(c) American Mathematical Society 1973 
is a locally convex algebra whose topology is defined via a family of $A$-convex seminorms \{multiples of submultiplicative seminorms\}. A basic reference to locally $m$-convex algebras is [5]. Examples of $A$-convex algebras (see [2]) include: (1) all locally $m$-convex algebras; (2) the space $\left(C_{b}(x), \beta\right)$ of bounded $C$-valued functions on a locally compact Hausdorff space with the strict topology; and (3) multiplier algebras.

We will denote by $x \circ y$ the operation on $E$ defined by $x \circ y=x+y-x y$. If $x \circ y=y \circ x=0$ then $x$ is called the quasi-inverse of $y$.

2. Algebraic properties. The Gel'fand-Mazur theorem forms the cornerstone of the basic representation theorem for Banach algebras [7]. Indeed, one of the important features of locally $m$-convex algebras is the fact that this theorem holds in this class of algebras [5]. Several authors (e.g. [5], [4], [6]) have exploited this type of representation theory, some obtaining results by assuming a sufficiently large carrier space (which the Gel'fand-Mazur theorem assures). We note here the fact that the Gel'fandMazur theorem holds for the larger class of $A$-convex algebras. Then we note several algebraic properties of these algebras, reducing to the $m$ convex and/or Banach case such questions as existence of identity, inverses and quasi-inverses.

TheOREM 2.1 (Gel'FAND-MAZUR). Every A-convex division algebra over $\boldsymbol{C}$ is isomorphic to the complex field $\boldsymbol{C}$.

Proof. This follows immediately from a remark of Williamson [13, p. 730].

It is of some interest to note the departure of the $m$-convex theory at this point. Theorem 2.1 for $m$-convex algebras is a direct transplant from the Banach algebra theorem. One simply notes that quasi-inversion is continuous for $m$-convexity. But a result of Turpin [8] shows that quasiinversion is never continuous in an $A$-convex algebra which is not $m$ convex. Thus $\left(C_{b}(R), \beta\right)$ gives an easy example of an algebra with quasiinversion not continuous.

In view of the representation theorem promised, we need the following simple consequences of Theorem 2.1:

THEOREM 2.2. Let E be a commutative complex A-convex algebra.

(i) If $M$ is a closed regular maximal ideal in $E$ then $E / M$ is isomorphic to $C$;

(ii) There is one-to-one correspondence between the set $\mathfrak{M}$ of nonzero continuous multiplicative linear functionals and the closed maximal regular ideals of $E$ in which a functional is associated with its kernel.

One of the main features of complete locally $m$-convex algebras is the theorem of Michael [5, Theorem 5.2] which reduces to Banach algebras 
the questions of existence of identity, inverses and quasi-inverses. Let $\boldsymbol{M}$ denote the category of locally $m$-convex algebras with continuous algebraic homomorphisms and, similarly, $\boldsymbol{A}$ the category of $A$-convex algebras. Warner [11] showed that $\dot{A}_{1}=\bigcup\left\{A^{n}: n=1,2, \cdots\right\}$ is the smallest idempotent subset of an algebra $E$ which contains $A$. Let $A^{*}$ denote the absolutely convex hull of $A_{1}$ for a given set $A$.

Proposition 2.3. Let $(E, \tau)$ be an A-convex algebra with $\mathscr{N}$ the neighborhood filter at zero. Then the filter $m(\mathscr{N})$, generated by all sets $V^{*}, V$ in $\mathcal{N}$, gives a locally $m$-convex topology on $E$ which is the finest locally m-convex topology on $E$ coarser than $\tau$. The correspondence thus described gives an idempotent functor $m: \boldsymbol{A} \rightarrow \boldsymbol{M}$.

Proof. The proof of the first part is given in [2]; the second part follows from the fact that if $f$ is an algebraic homomorphism then $\left[f\left(A^{*}\right)\right] \subset$ $[f(A)]^{*}$ and similar straightforward calculations.

By using the functor $m$ of Proposition 2.3 the problems of determining algebraic properties of an $A$-convex algebra are reduced to the locally $m$-convex case. The filter $m(\mathscr{N})$ is defined for any locally convex algebra so $m$ can be considered as a functor on all such algebras.

Proposition 2.4. Let $E$ be an A-convex algebra, $m(E)$ a complete Hausdorff algebra. Then the existence of identity, inverses and quasiinverses is equivalent to their existence in a set of Banach algebras.

Proof. Clearly, existence of identity, inverses and quasi-inverses is independent of the topology on $E$. Hence Michael [5, Theorem 5.2] gives the result.

EXAMPLE 2.5. The functor $m$ associates $\left(C_{b}(X), \kappa\right)$ with the space $\left(C_{b}(X), \beta\right)$. This is shown in [2] so that there are no locally $m$-convex topologies on $C_{b}(X)$ between the compact-open and the strict.

THEOREM 2.6. For any locally convex algebra E, the set of continuous multiplicative linear functionals is the same for both $E$ and $m(E)$.

Proof. A linear functional is continuous if and only if it is bounded on a neighborhood of zero. Thus, if $m$ is a multiplicative linear functional which is bounded on a neighborhood $V$ in $E$ then for a suitable multiple $W$ of $V, m(W) \leqq 1$. Since $m$ is multiplicative $m\left(W^{*}\right) \leqq 1$ so $m$ is continuous in $m(E)$. Since the topology of $m(E)$ is coarser than the topology of $E$, the result follows. We now consider the question of when $m(E)$ is Hausdorff and/or complete.

Definition 2.7. For any locally convex algebra $E$ with local neighborhood filter $\mathscr{N}$, an element $x \in E$ is topologically nilpotent if $x$ is an element of $V_{0}=\bigcap\left\{V^{*}: V \in \mathscr{N}\right\}$. Denote the set of topological nilpotents by $\mathscr{E}$. 
LeMma 2.8. Let E be a locally convex algebra. The subspace is precisely the closure of $(0)$ in $m(E)$ and contains all $x \in E$ such that $x^{n}$ converges to 0 as $n \rightarrow \infty$.

Proof. Immediate from the definition of $m$ and the fact that the closure of $(0)$ is the intersection of all local neighborhoods.

THEOREM 2.9. Let $E$ be a locally convex algebra. Then $m(E)$ is Hausdorff if and only if $\mathscr{E}=(0)$. Further, $\mathscr{E}$ is closed with respect to quasi-inversion.

Proof. A locally convex space is closed if and only if $\operatorname{cl}(0)=(0)$ so Lemma 2.8 gives the first part. The second part is an easy consequence of the definition.

The result of Theorem 2.9 is that quasi-inverses may be investigated by checking the space $\mathscr{E}$ together with the Hausdorff $m$-convex algebra $m(E) / \mathscr{E}$.

There is no relation between completeness of $E$ and completeness of $m(E)$. Using Example $2.5,\left(C_{b}(\boldsymbol{R}), \beta\right)$ is complete but $\left(C_{b}(\boldsymbol{R}), \boldsymbol{\kappa}\right)$ is not complete. Wang [10] gives an example where both $\beta$ and $\kappa$ are complete. The $A$-normed algebra given in [1] has the property that neither $E$ nor $m(E)$ (the compact-open on $C_{b}(0,1)$ ) is complete. However, for function spaces the determination of completeness is often easily checked.

Many of the $m$-convex consequences of Theorem 5.2 of [5] have immediate analogues for the $A$-convex setting which are not included here.

3. Representation. Morris and Wulbert [6] gave a general situation for representation of a commutative locally convex algebra as a subspace of some $(C(X), \kappa)$ where $X$ is at least completely regular and $\kappa$ denotes the compact-open topology. Since $\kappa$ always gives a locally $m$-convex topology, there is no hope of a satisfactory representation theorem in the $A$-convex case. For a subclass of $A$-convex algebras, we provide a suitable representation theorem here. As a consequence, the relations expressed by A. Mallios [4] in terms of " $m$-barrelled" can be viewed in a different way. The fact that $m$-barrelled $A$-convex algebras (see [1]) are $m$-convex provides the bridge between our theorem and some of the results of [4] and [6].

Let $E$ be a commutative $A$-convex algebra and $\mathfrak{M}$ the space of nonzero continuous multiplicative linear functionals topologized via the $\sigma\left(E^{\prime}, E\right)$ topology. Theorem 2.2 gives the usual relationship with maximal ideals. Let $G$ denote the Gel'fand map of $E \rightarrow C(\mathfrak{M})$ given by $G(x)(m)=m(x)=$ $\hat{x}(m)$. Then $G$ gives an algebraic isomorphism if and only if $E$ is semisimple. For $A$-convex algebras, semisimple is equivalent to "strongly semisimple" as defined in [6]. 
Definition 3.1. A locally convex algebra $E$ is uniformly $A$-convex if there is some defining family of seminorms $\left\{p_{\alpha}: \alpha \in A\right\}$ with the property that for each $x$ in $E$ there are constants $M_{x}$ and $N_{x}$ such that

$$
p_{\alpha}(x y) \leqq M_{x} p_{\alpha}(y) \text { and } p_{\alpha}(y x) \leqq N_{x} p_{\alpha}(y)
$$

for all $\alpha \in A$ and for all $y \in E$. (Such a family will be called a uniform family.)

Clearly, uniformly $A$-convex algebras are $A$-convex. Examples include $\left(C_{b}(X), \beta\right),\left(C_{b}(X), \kappa\right)$ and $A$-normed [3] algebras. The space $(C(X), \kappa)$ is an $m$-convex algebra which is not, in general, uniformly $A$-convex (this last fact can be seen from the sequel).

Let $E$ be a commutative $A$-convex algebra with identity $e$. Then a uniform defining family $\left\{p_{\alpha}: \alpha \in A\right\}$ can be chosen such that $p_{\alpha}(e)=1$ for all $\alpha \in A$. If $E$ does not have an identity, a uniform family can be chosen with this property on $E^{+}$, the $A$-convex algebra obtained by adjoining an identity. We assume such a family is always chosen in the following results. Define

$$
\|x\|=\inf \left\{M_{x}: p_{\alpha}(x y) \leqq M_{x} p_{\alpha}(y), \text { for all } \alpha \in A, y \in E\right\} .
$$

Then it is easy to verify that

$$
\|x\|=\sup \left[\sup \left\{p_{\alpha}(x y): p_{\alpha}(y) \leqq 1\right\}: \alpha \in A\right] .
$$

LEMMA 3.2. The norm defined by $\left(^{*}\right)$ is a submultiplicative norm on $E$ for which $p_{\alpha}(x) \leqq\|x\|$ for all $\alpha \in A, x \in E$.

PROOF. This result is obtained by using (**) and properties of suprema. If $E$ has an identity, $p_{\alpha}(x)=p_{\alpha}(x e) \leqq M_{x} p_{\alpha}(e)=M_{x}$ for all $\alpha$, and $\|x\|$ is the inf of all such constants $M_{x}$ so $p_{\alpha}(x) \leqq\|x\|$. If $E$ does not have an identity, one can be adjoined and the above argument can be applied.

Let $E_{n}$ denote the normed space $(E,\|\|)$ and $\mathscr{M}_{n}$ the carrier space of $E_{n}$. The respective dual spaces are related by $E_{n} \supset E^{\prime}$ and the structure spaces by $\mathfrak{M}_{n} \supset \mathfrak{M}$. If $E_{n}$ is complete we have

TheOREM 3.3. The structure space $\mathfrak{M}$ is locally compact and the Gel'fand transform of $x$ in $E$ is the restriction of the Gel'fand transform of $x$ in $E_{n}$. $\mathfrak{M}$ is Hausdorff if and only if $E$ is semisimple.

Proof. Since $\mathfrak{M}_{n}$ is locally compact [7] and $\mathfrak{M}$ is a closed subset, $\mathfrak{M}$ is locally compact. The remainder of the theorem is clear.

For each $\alpha \in A$, define the extended real number

$$
t_{\alpha}(m)=\sup \left\{|\hat{x}(m)|: p_{\alpha}(x) \leqq 1\right\} .
$$

Then define $\phi_{\alpha}: \mathfrak{M} \rightarrow R_{+}$by $\phi_{\alpha}(m)=0$, if $t_{\alpha}(m)=+\infty$, and $\phi_{\alpha}(m)=1 / t_{\alpha}(m)$ otherwise. 
Note that if $E$ has an identity then $0 \leqq \phi_{\alpha}(m) \leqq 1$ since $\hat{e}(m)=1$ gives $t_{\alpha}(m) \geqq 1$. Let $H$ be the subspace of $C(\mathfrak{M})$ defined by

$$
H=\left\{f \in C(\mathfrak{M}): \phi_{\alpha} f \text { is bounded for all } \alpha \in A\right\} .
$$

Clearly $C_{b}(\mathfrak{M}) \subset H$.

THEOREM 3.4. Let $E$ be a commutative uniformly A-convex algebra with Gel'fand map $G$. Then $G(E) \subset C_{b}(\mathfrak{M}) \subset H$.

Proof. Since $|\hat{x}(m)|=|m(x)| \leqq\|x\|, G(E) \subseteq C_{b}(\mathfrak{M})$.

Let $\beta$ denote the topology on $H$ defined by the family of seminorms $\left\{\hat{p}_{\alpha}: \alpha \in A\right\}$ where

$$
\hat{p}_{\alpha}(f)=\sup \left\{\left|f(m) \phi_{\alpha}(m)\right|: m \in \mathfrak{M}\right\}, \quad f \in H .
$$

This gives a type of strict topology on $H$ replacing the compact-open in [4].

Theorem 3.5. Let $E$ be uniformly A-convex and $G$ the Gel'fand transform. Then $G$ is continuous from $E$ into $(H, \beta)$. If $E$ is semisimple then $E$ is represented via the continuous isomorphism $G$.

PRoof. If $p_{\alpha}(x) \neq 0$, let $y=\left[p_{\alpha}(x)\right]^{-1} x$. Then $p_{\alpha}(y)=1$ and $m(x)=$ $p_{\alpha}(x) m(y)$. From the definition of $\hat{p}_{\alpha}(\hat{x}), \hat{p}_{\alpha}(\hat{x}) \leqq p_{\alpha}(x) \sup \left\{|m(y)| / t_{\alpha}(m)\right\} \leqq$ $p_{\alpha}(x)$. If $p_{\alpha}(x)=0$ then $p_{\alpha}(k x)=0$ for every natural number $k$. Hence $t_{\alpha}(m)=+\infty, \phi_{\alpha}(m)=0$ and $\hat{p}_{\alpha}(\hat{x})=0 \leqq p_{\alpha}(x)$. Thus, $\hat{p}_{\alpha}(\hat{x}) \leqq p_{\alpha}(x)$ for all $x \in E$ so $G$ is continuous.

REMARK 3.6. The seminorms $p$ can often be defined in more general cases, and Theorem 3.5 still holds with the same proof. We note also that if all the $\phi_{\alpha}$ vanish at $\infty$ and generate $C_{0}(\mathfrak{M})$ then we get the strict topology. If there is but one $\phi_{\alpha}$ which is bounded away from zero we get the normed case. Thus, the representation theorem given here is general enough to include previous results.

Definition 3.7. A commutative uniformly $A$-convex algebra is saturated if for a uniform defining family $\left\{p_{\alpha}: \alpha \in A\right\}$ the following property holds for each $p_{\alpha}$ : for each $x$ in $E$ such that $p_{\alpha}(x)=1$ there exists $m_{0} \in \mathfrak{M}$ such that $m_{0}(x)=\sup \left\{|\hat{y}(m)|: p_{\alpha}(y) \leqq 1\right\}$ for some $m \in \mathfrak{M}$. (Then $\hat{p}_{\alpha}(\hat{x})=1=p_{\alpha}(x)$.)

THEOREM 3.8. If $E$ is a commutative saturated uniformly A-convex algebra then $G$ is an open map. Hence if $E$ is semisimple, $G$ is a topological and algebraic isomorphism.

Proof. If $E$ is saturated then $\hat{p}_{\alpha}(\hat{x})=p_{\alpha}(x)$ for all $x \in E$ and $\alpha \in A$. Hence if $E$ is semisimple $G$ is a bijection onto $G(E)$ with $G$ and $G^{-1}$ continuous. 
REMARK 3.9. If $G$ is an algebraic and topological isomorphism and $E$ is barrelled (or $i$-barrelled [11] and [4]) then $E$ is locally $m$-convex. This gives a connection to the results in [4] and those of this section. In any case, with the conditions of Theorem 3.5 we may apply the functor $m$ of $\$ 2$ to obtain a representation in a locally $m$-convex algebra. Since application of $m$ gives a coarser topology, $G$ is still continuous. Of course, if $G(E)$ is $\left(C_{b}(X), \beta\right)$ then this gives $\left(C_{b}(X), \kappa\right)$.

\section{REFERENCES}

1. A. C. Cochran, R. Keown and C. R. Williams, On a class of topological algebras, Pacific J. Math. 34 (1970), 17-25. MR 42 \#8278.

2. A. C. Cochran, Topological algebras and Mackey topologies, Proc. Amer. Math. Soc. 30 (1971), 115-119. MR 45 \#897.

3. - Inductive limits of A-convex algebras, Proc. Amer. Math. Soc. 37 (1973), 489-496.

4. A. Mallios, On functional representations of topological algebras, J. Functional Analysis 6 (1970), 468-480. MR 42 \#5047.

5. E. A. Michael, Locally multiplicatively-convex algebras, Mem. Amer. Math. Soc. no. 11 (1952). MR 14, 482.

6. P. D. Morris and D. E. Wulbert, Functional representation of topological algebras, Pacific J. Math. 22 (1967), 323-337. MR 35 \#4730.

7. M. A. Naìmark, Normed rings, rev. ed., GITTL, Moscow, 1956; English transl., Noordhoff, Groningen, 1964. MR 19, 870; 34 \#4928.

8. P. Turpin, Une remarque sur les algebres d̀ inverse continu, C. R. Acad. Sci. Paris Sér. A-B 270 (1970), A1686-A1689. MR 42 \#3571.

9. L. Waelbrock, Topological vector spaces and algebras, Lecture Notes in Math., no. 230, Springer-Verlag, Berlin and New York, 1971.

10. J. Wang, Multipliers of commutative Banach algebras, Pacific J. Math. 11 (1961), 1131-1149. MR 25.\#1462.

11. S. Warner, Inductive limits of normed algebras, Trans. Amer. Math. Soc. 82 (1956), 190-216. MR 18, 52.

12. - The topology of compact convergence on continuous function spaces, Duke Math. J. 25 (1958), 265-282. MR 21 \#1521.

13. J. H. Williamson, On topologizing the field $C(t)$, Proc. Amer. Math. Soc. 5 (1954), 729-734. MR 16, 145.

14. W. Zelazko, On generalized topological divisors of zero in m-convex locally convex algebras, Studia Math. 28 (1966), 9-16. MR 34 \#3362.

Department of Mathematics, Duke University, Durham, North Carolina 27706

Department of Mathematics, University of Arkansas, FayetteVille, Arkansas 72701 